\title{
The MMP-1/PAR-1 Axis Enhances Proliferation and Neuronal Differentiation of Adult Hippocampal Neural Progenitor Cells
}

\author{
Maria Maddalena Valente, ${ }^{1}$ Megan Allen, ${ }^{2}$ Valeria Bortolotto, ${ }^{1}$ Seung T. Lim, \\ Katherine Conant, ${ }^{2}$ and Mariagrazia Grilli ${ }^{1}$ \\ ${ }^{1}$ Laboratory of Neuroplasticity, Department of Pharmaceutical Sciences, University of Piemonte Orientale "Amedeo Avogadro", \\ 28100 Novara, Italy \\ ${ }^{2}$ Department of Neuroscience, Georgetown University Medical Center, Washington, DC 20057, USA
}

Correspondence should be addressed to Katherine Conant; kec84@georgetown.edu and Mariagrazia Grilli; mariagrazia.grilli@uniupo.it

Received 19 June 2015; Revised 13 August 2015; Accepted 6 September 2015

Academic Editor: Fabienne Agasse

Copyright (C) 2015 Maria Maddalena Valente et al. This is an open access article distributed under the Creative Commons Attribution License, which permits unrestricted use, distribution, and reproduction in any medium, provided the original work is properly cited.

\begin{abstract}
Matrix metalloproteinases (MMPs) are zinc-dependent endopeptidases that play a role in varied forms of developmental and postnatal neuroplasticity. MMP substrates include protease-activated receptor-1 (PAR-1), a G-protein coupled receptor expressed in hippocampus. We examined proliferation and differentiation of adult neural progenitor cells (aNPCs) from hippocampi of mice that overexpress the potent PAR-1 agonist MMP-1. We found that, as compared to aNPCs from littermate controls, MMP- 1 tg aNPCs display enhanced proliferation. Under differentiating conditions, these cells give rise to a higher percentage of MAP- $2^{+}$neurons and a reduced number of oligodendrocyte precursors, and no change in the number of astrocytes. The fact that these results are MMP and PAR-1 dependent is supported by studies with distinct antagonists. Moreover, JSH-23, an inhibitor of NF- $\kappa$ B p65 nuclear translocation, counteracted both the proliferation and differentiation changes seen in MMP-1 tg-derived NPCs. In complementary studies, we found that the percentage of Sox $2^{+}$undifferentiated progenitor cells is increased in hippocampi of MMP- 1 tg animals, compared to wt mice. Together, these results add to a growing body of data suggesting that MMPs are effectors of hippocampal neuroplasticity in the adult CNS and that the MMP-1/PAR-1 axis may play a role in neurogenesis following physiological and/or pathological stimuli.
\end{abstract}

\section{Introduction}

Newly born neurons that are generated in the dentate gyrus (DG) of the adult hippocampus are increasingly appreciated as potential effectors of cognitive flexibility, pattern separation, and spatially precise search strategies [1-3]. Dysregulated adult hippocampal neurogenesis (AHN) is a correlate of varied physiological and pathological states, and may contribute to and/or result from the same. While adult neurogenesis can be modulated by numerous stimuli, several conditions characterized by an increase in progenitor cell proliferation are coincidently associated with an increase in the level and activity of secreted enzymes belonging to the matrix metalloproteinase (MMP) family [4-6]. Prior studies have also implicated MMP activity in the proliferation of varied cell types including neural progenitors [7], but the overall role of specific MMPs in aNPC proliferation and the mechanism(s) or substrates important to MMP-dependent effects are not particularly well understood.

Potential protease substrates that are highly expressed within the adult hippocampus include synaptic cell adhesion molecules and protease-activated receptor-1 (PAR-1), a GPCR activated by cleavage of $\mathrm{N}$-terminal sequence which in turn exposes a tethered peptide ligand [8-10]. PAR-1 is of particular interest to neurogenesis since it is expressed in the DG and found on both neurons and glial cells $[8,11]$. Moreover, its signaling has been linked to increases in the function of NMDARs thought to play a role in neurogenesis as well as to increased release of soluble factors that enhance progenitor cell proliferation $[8,12,13]$. 
PAR-1 activators include thrombin and APC, two bloodderived proteases that may enter the CNS in response to blood brain barrier damage as well as a small subset of MMPs (MMP-1 and MMP-13) [9, 14, 15]. MMP-1 in particular is equipotent to thrombin in its ability to stimulate receptor cleavage and PAR-1 dependent intracellular signaling [14]. Of note, however, is the fact that MMP-1 cleavage of PAR1 generates a relatively unique tethered peptide ligand since cleavage occurs as a site that is two amino acids N-terminal to the thrombin cleavage site [15].

MMP-1 has the potential to be an important PAR-1 agonist in the absence of blood brain barrier damage. It is expressed by astrocytes [16], and while basal expression is typically low, levels of MMP-1 may be increased in association with physiological and pathological stimuli that are linked to neurogenesis [17-19]. MMP-1 dependent activation of PAR-1 has, however, not been well explored with respect to its function in central nervous system physiology or neurogenesis in particular.

Quiescent neural stem/progenitor cells, thought to generate neurons that will ultimately mature, express markers including GFAP [20]. In the present study, we therefore take advantage of a unique mouse model in which human MMP-1 is expressed under the control of a GFAP promoter to examine the MMP-1/PAR-1 axis in adult hippocampal neurogenesis. Human MMP-1 is an orthologue of the much later identified murine MMP-1a [21], and, importantly, it activates murine PAR-1 [22]. We show that the MMP-1/PAR1 axis can increase both progenitor cell proliferation and neuronal fate specification and we suggest that future studies are warranted to determine if this axis is important to progenitor cell plasticity in physiological settings such as exercise and environmental enrichment as well as in pathological conditions.

\section{Materials and Methods}

2.1. Animals. Adult male (3-4-month-old) wild type C57/BL6 or heterozygous littermate MMP-1 tg mice on a C57/BL6 background were used in the present study. The MMP-1 tg animals were prepared at the Johns Hopkins University via pronuclear injection of a glial fibrillary acid protein- (GFAP-) MMP-1 construct. This construct encodes human MMP-1, an orthologue of the much later identified mouse MMP-1a [21]. Importantly, human MMP-1 cleaves murine PAR-1 [22]. We obtained the MMP-1 construct from Dr. Jeanine D'Armiento who has used it to overexpress MMP-1 in mouse's heart and lung $[23,24]$. The generation of the combined GFAP-MMP1 construct was carried out using a GFAP promoter from Dr. Michael Brenner [25]. All experimental procedures were performed with approval of the Johns Hopkins University and Georgetown University Animal Care and Use Committee guidelines.

2.2. Isolation and Culture of Adult Neural Progenitor Cells (aNPCs) from Murine Hippocampus. For each aNPC preparation, three adult (3-4-month-old) male mice were humanely euthanized. The brains were extracted and hippocampi were isolated under a dissecting microscope using fine surgical instruments and collected in ice-cold PIPES buffer $\mathrm{pH} 7.4$ containing $20 \mathrm{mM}$ PIPES, $25 \mathrm{mM}$ glucose, $0.5 \mathrm{M} \mathrm{KCl}$, $0.12 \mathrm{M} \mathrm{NaCl}$ (Sigma, St. Louis, MO), and $100 \mathrm{U} / 100 \mu \mathrm{g} / \mathrm{mL}$ Penicillin/Streptomycin solution (Invitrogen, Carlsbad, CA). After centrifugation $(110 \times \mathrm{g} \times 5 \mathrm{~min})$, tissue was digested for 40 minutes at $37^{\circ} \mathrm{C}$ using the Papain Dissociation System (Worthington DBA, Lakewood, NJ). The cell suspension was placed into $25 \mathrm{~cm}^{2}$ Falcon cell-culture flasks (Thermo Fisher Scientific, Rockville, MD) and cultured in growth medium [Neurobasal-A medium containing B27 supplement, $2 \mathrm{mM}$ L-glutamine (Invitrogen), $10 \mathrm{ng} / \mathrm{mL}$ human basic fibroblast growth factor (bFGF-2, PeproTech, Rocky Hill, NJ), $20 \mathrm{ng} / \mathrm{mL}$ human epidermal growth factor (EGF, Sigma), $4 \mu \mathrm{g} / \mathrm{mL}$ heparin sodium salt (Sigma), and $100 \mathrm{U} / 100 \mu \mathrm{g} / \mathrm{mL}$ Penicillin/Streptomycin]. Primary (Passage 1, P1) neurospheres were passaged after 7-9 days in vitro (DIV), whereas subsequent passages were prepared every 5 DIV. At each passage, cells were plated in a T25 flask at a density of 12,000 cells $/ \mathrm{cm}^{2}$ in growth medium. P3-P10 neurospheres were utilized for experiments.

2.3. aNPC Differentiation and Proliferation. For evaluation of cell proliferation, dissociated aNPCs were plated onto LuminNUNC F96 MicroWell plates (Thermo Fisher Scientific), at a density of 4,000 cells per well, in growth medium in presence of $1 \mu \mathrm{M}$ BMS-200261, $8 \mu \mathrm{M}$ MMP inhibitor II, $3 \mu \mathrm{M}$ JSH-23 (Calbiochem, Merck KGaA, Darmstadt, Germany), or vehicle for 0 to $72 \mathrm{~h}$, as indicated. Proliferation rates were determined by using the CellTiter-Glo Luminescent Cell Viability Assay (Promega, Madison, WI), according to the manufacturer's instructions. All experiments were performed in triplicate, and data (expressed as counts per second) represent mean \pm standard deviation (SD), as indicated.

For differentiation experiments, we used a previously described protocol [26]. Briefly, neurospheres were dissociated and plated onto laminin-coated chamber slides in Neurobasal-A medium with B27 supplement and $2 \mathrm{mM}$ L-glutamine. At plating, NPCs were treated with vehicle, $1 \mu \mathrm{M}$ of the PAR-1 antagonist BMS-200261 trifluoroacetate (Sigma), or $3 \mu \mathrm{M}$ JSH-23 (Calbiochem). After $24 \mathrm{~h}$, cells were washed in PBS and fixed with ice-cold 4\% PFA for $20 \mathrm{~min}$ at RT for subsequent immunofluorescence analysis. In each experiment, 5 fields/well (corresponding to 100150 cells/well) were evaluated. All experiments were run in triplicate, and experiments were repeated at least three times. Data represent the mean value \pm SD as noted. In parallel, the survival rate in culture was evaluated in each fixed culture as previously described [26].

2.4. Immunocytochemical Analysis. After fixation, neurosphere-derived differentiated cells were washed three times in PBS and permeabilized in PBS containing $0.48 \%$ (vol/vol) Triton X-100 (Sigma) for $5 \mathrm{~min}$ at RT. The primary antibodies against MAP-2 (rabbit polyclonal, 1:600, Chemicon, Temecula, CA), GFAP (mouse monoclonal, 1:600, Millipore, Billerica, MA), or NG2 (rabbit polyclonal, 1:500, Abcam, Cambridge, MA) were incubated for $150 \mathrm{~min}$ at RT in an antibody solution containing 16\% (vol/vol) goat 
serum. Secondary antibodies were as follows: Alexa Fluor 488 conjugated goat anti-rabbit antibody (1:1600, Invitrogen) and Alexa Fluor 488 conjugated goat anti-mouse antibody (1:1600, Invitrogen). Nuclei were counterstained with DAPI (1:10000, Sigma). Fluorescent Mounting Medium (Electron Microscopy Sciences, Hatfield, PA) was applied to slides as antifading agent prior to addition of coverslips.

2.5. Protein Isolation and Western Blot Analysis. For protein isolation, aNPC were lysed in 3x vol/vol of ice-cold hypotonic RIPA buffer [50 mM Tris- $\mathrm{HCl}$ pH 7.5, $150 \mathrm{mM} \mathrm{NaCl}, 0.5 \mathrm{mM}$ EDTA pH 8, 1\% (vol/vol) Triton X-100, 0.1\% (wt/vol) SDS, $1 \mathrm{mM}$ DTT, protease inhibitor mix, and phosphatase inhibitor cocktails 2 and 3 (Sigma)] for 25 min on ice. Lysates were centrifuged at $1,300 \times \mathrm{g}$ for $10 \mathrm{~min}$ at $4^{\circ} \mathrm{C}$ and supernatants were collected. Protein concentration was determined by Pierce BCA protein assay kit (Pierce, Rockford, IL) and equivalent amounts of each sample were separated onto SDS-PAGE gel and transferred onto nitrocellulose membranes. Molecular weights were inferred by comparison to prestained markers (BioRad, Hercules, CA). Membranes were then blocked in $5 \%$ nonfat dry milk in phosphate-buffered saline (PBS) with $0.1 \%$ Tween (PBST) for $1 \mathrm{~h}$. The blot was subsequently probed with a primary antibody to PAR-1 (H-111, 1:500, Santa Cruz Biotechnology, Santa Cruz, CA). After washing the membrane in PBST, it was incubated with an appropriate secondary antibody. The membrane was then washed again in PBST and immunoreactive bands were visualized using electrochemiluminescence (Amersham Pharmacia Biotech, Piscataway, NJ, USA).

2.6. ELISA. ELISA for total MMP-1 was performed on neurosphere supernatants using a commercially available kit (R\&D Systems, Minneapolis, MN) according to the manufacturer's instructions.

2.7. Flow Cytometry. Animals were euthanized with $\mathrm{CO}_{2}$ intoxication and immediately perfused with PBS. For each experiment, two hippocampi from littermates of the same genotype (WT, $n=2$; hMMP-1 tg, $n=2$ ) were microdissected and combined in a $15 \mathrm{~mL}$ conical tube filled with $10 \mathrm{~mL}$ of ice-cold dissection medium $\left(\mathrm{Ca}^{2+}\right.$ and $\mathrm{Mg}^{+}$free HBSS, $0.01 \% 100 \mathrm{mM}$ Sodium Pyruvate, $0.10 \%$ Glucose, and $0.01 \% 1 \mathrm{M}$ HEPES) and gently washed 2 times. Dissection medium was aspirated and tissues were incubated in $5 \mathrm{~mL}$ of $0.25 \%$ Trypsin in HBSS plus phenol red (Invitrogen) at $37^{\circ} \mathrm{C}$ for 10 minutes. Next, $0.5 \mathrm{~mL}$ of $1 \%$ DNase solution was added to tube and incubated on bench for 3 minutes. This volume was aspirated and tissue was gently washed 3 times in temperature equilibrated plating medium (MEM with Earle's BSS, 10\% FBS, $0.45 \%$ glucose, $0.01 \% 100 \mathrm{mM}$ Sodium Pyruvate, 0.01\% $200 \mathrm{mM}$ Glutamine, and 0.01 Penicillin/Streptomycin). Tissues were then triturated in $2 \mathrm{~mL}$ total volume of plating medium followed by filtration through a $70 \mu \mathrm{m}$ pore. Cell suspension was gently centrifuged and supernatant discarded. Next, cells were resuspended in $4 \%$ paraformaldehyde/PBS for $15 \mathrm{~min}$ with gentle rotation at room temperature. After one wash and centrifugation in
PBS, cell membranes were permeabilized in $100 \% \mathrm{MeOH}$ for $10 \mathrm{~min}$ at $4^{\circ} \mathrm{C}$. Cells were washed 3 times in PBS and incubated overnight in antibody solution (Phycoerythrin (PE) conjugated anti-SOX2; 656103, BioLegend, San Diego, CA) made up in PBS-TX at $4^{\circ} \mathrm{C}$. An aliquot of cells was used as autofluorescence control. Incubations were performed at $4^{\circ} \mathrm{C}$ overnight. Flow cytometric analysis was performed with a BD FACS Aria III machine and FCS express software by De Novo. For each of the gated populations the percentage and geometric mean fluorescence intensity (MFI) were analyzed.

2.8. Statistical Analysis. For all experiments using ex vivo aNPCs data are expressed as mean \pm SD or SE, as indicated, of at least three experiments in triplicate. Data were analyzed by two-tailed, unpaired, Student's $t$-test for comparison of two groups or ANOVA, followed by Tukey's post hoc analysis, for comparison of groups greater than two. Statistical significance level was set for $p$ values less than or equal to 0.05 .

\section{Results}

3.1. aNPCs from MMP-1 tg Animals Show Increased Proliferation. NPCs were harvested and cultured from wild type and MMP-1 tg animals. As shown in Figure 1(a), supernatants from tg cultures express hMMP-1. Cell proliferation was subsequently observed as a function of time in culture in WT-derived and tg-derived cells. Interestingly, aNPCs from tg animals showed enhanced proliferation, when compared to WT aNPC, at 24, 48, and $72 \mathrm{~h}$ time points (Figure 1(b)). Results shown are mean \pm SD from four replicate wells per genotype and are representative of an experiment performed with different NPC preparations on three separate occasions $\left({ }^{*} p<0.05 ;{ }^{* *} p<0.01\right)$. The fold increase from WT control $(0 \mathrm{~h})$ is shown (baseline CPS values were 51,881 $\pm 10,394$ for WT and 51,422 \pm 10,473 for MMP-1 tg). Consistent with increased proliferation as opposed to survival, as determined by published techniques [26], cell viability in WT and tg cultures did not differ (mean percentage \pm SD of viable cells: WT: $88.8 \pm 2.3, n=9$; MMP-1 tg: $89.2 \pm 1.02$, Student's $t$-test).

\subsection{MMP-1 tg-Derived aNPCs Show Increased Differentiation} towards the Neuronal Lineage. To address the question of whether NPC differentiation is altered in the background of enhanced MMP-1 expression, we also performed differentiation experiments on aNPCs from WT and MMP-1 tg animals. Under appropriate conditions (removal of growth factors) NPCs express their multipotentiality and give rise to cells belonging to three neural lineages, namely, neurons, astrocyte, and oligodendrocyte precursors. Results (mean \pm SD), as shown in Figure 2(a), suggest that differentiation towards a neuronal lineage is enhanced in the setting of excess hMMP-1. We indeed observed a statistically significant increase in the percentage of MAP- $2^{+}$cells generated from hMMP-1 tg-derived compared to WT-derived aNPC $\left({ }^{* *} p<\right.$ 0.01 , Student's $t$-test). Interestingly, this effect was selective on the neuronal fate of MMP-1 tg NPC, since the number of $\mathrm{GFAP}^{+}$astrocytes was not different in the two genotypes, while the percentage of $\mathrm{NG}^{+}$oligodendrocyte precursors 

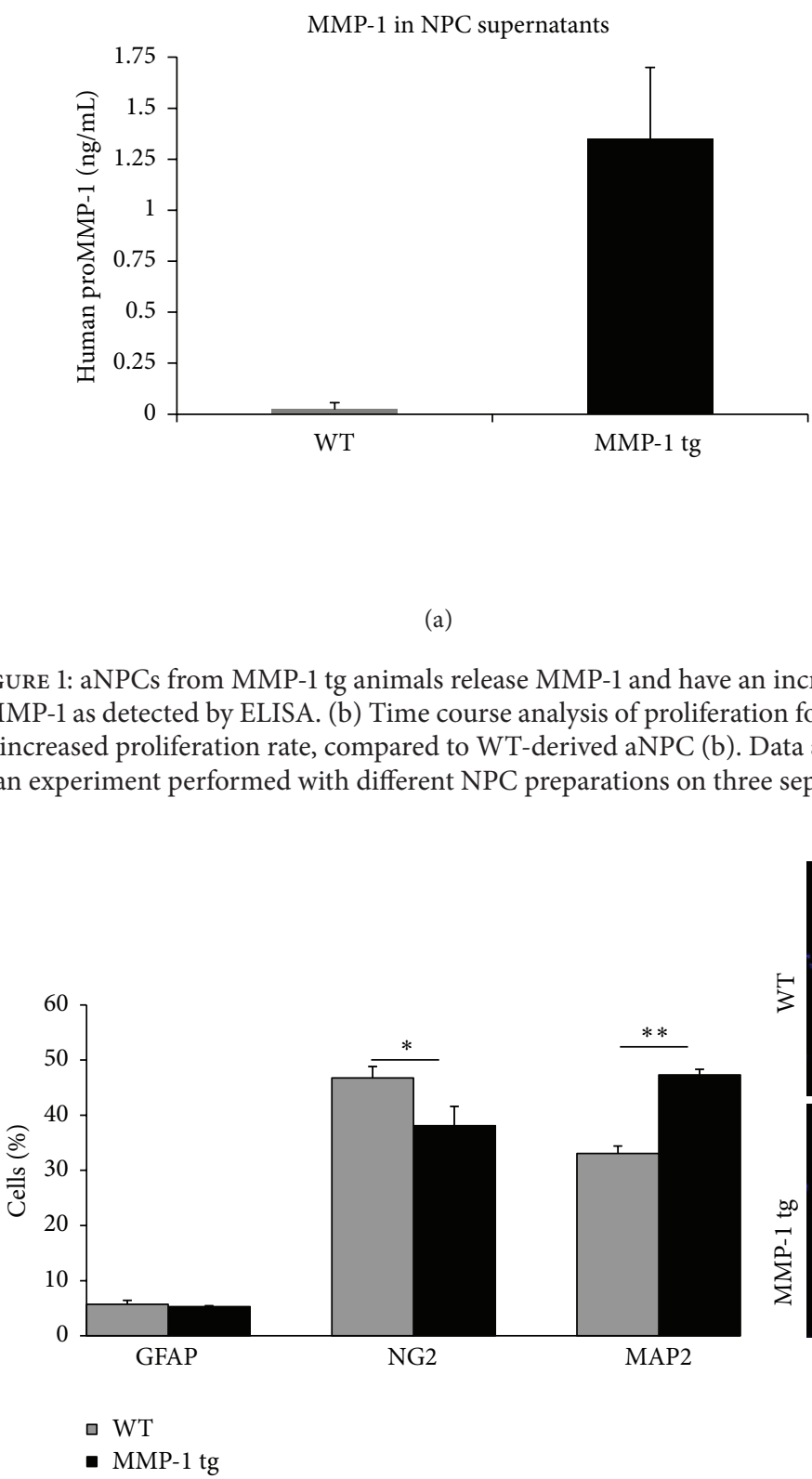

(a)

- MMP-1 tg

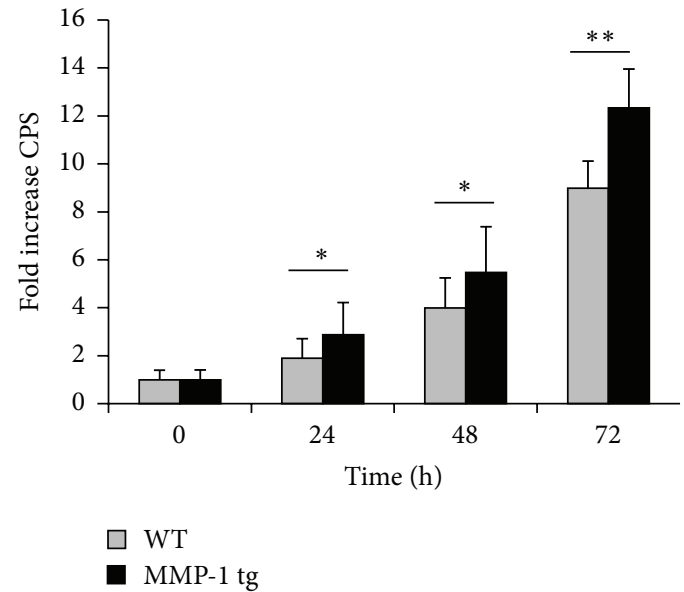

(b)

FIGURE 1: aNPCs from MMP-1 tg animals release MMP-1 and have an increased proliferation rate. (a) Supernatants from tg cultures express hMMP-1 as detected by ELISA. (b) Time course analysis of proliferation for WT and MMP-1 tg-derived aNPC. Cells from tg animals display an increased proliferation rate, compared to WT-derived aNPC (b). Data are the mean \pm SD from four replicate wells and are representative of an experiment performed with different NPC preparations on three separate occasions $\left({ }^{*} p<0.05 ;{ }^{* *} p<0.01\right.$ versus WT).
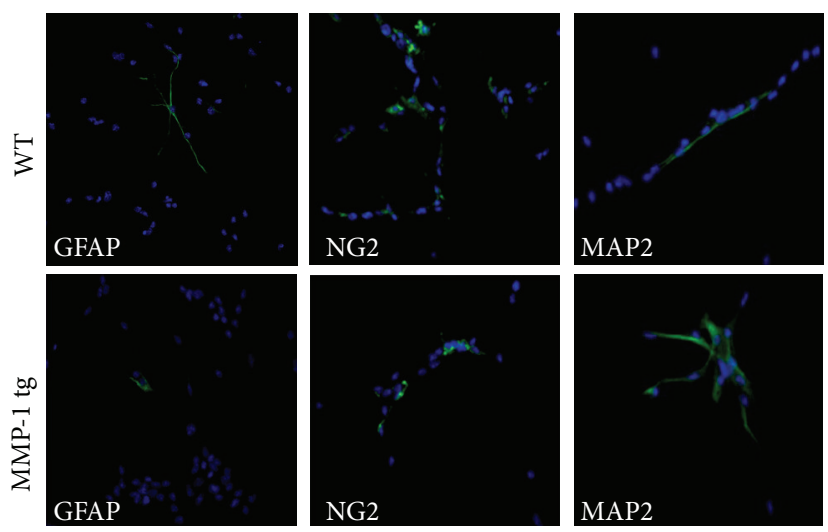

FiguRE 2: MMP-1 tg-derived aNPCs show increased differentiation towards the neuronal lineage. (a) Differentiation experiments on aNPCs from WT and MMP-1 tg animals. Data, which represent mean \pm SD values, are expressed as percentage over total number of viable cells. A statistically significant increased percentage of MAP- $2^{+}$neurons was generated in vitro by aNPCs from hMMP-1 tg, compared to WT counterpart. Conversely, the number of cells expressing the oligodendrocyte precursor marker NG2 is significantly reduced in absence of changes in the number of GFAP ${ }^{+}$cells in tg cultures $\left({ }^{*} p<0.05 ;{ }^{* *} p<0.01\right.$ versus WT cells, ANOVA). (b) Representative GFAP, NG2, and MAP2 immunostaining (in green) in WT and hMMP-1 tg NPC cultures. Nuclei are counterstained by DAPI (in blue).

was significantly reduced in tg-derived cultures compared to WT-derived cells ( ${ }^{*} p<0.05$, Student's $t$-test). Of note, there was no genotype effect on the percentage of apoptotic cells in the variously stained preparations (data not shown). Representative immunostaining for MAP2, GFAP, and NG2 in WT and hMMP-1 tg cultures is shown in Figure 2(b). Differentiation Are PAR-1 Dependent. PAR-1 is a GPCR that is activated by proteolytic cleavage within N-terminal domain. The receptor is expressed on neurons and glia in several brain regions (including hippocampus) $[8,27]$ and on NPC in the subventricular zone [28]. To determine whether PAR1 is expressed in NPC preparations from murine dentate gyrus, we performed a Western blot on cell lysates, using murine hippocampus as a control. As shown in Figure 3(a), a single predominant band of the expected molecular weight size $(46 \mathrm{kDa})$ was detected in both samples. To confirm 


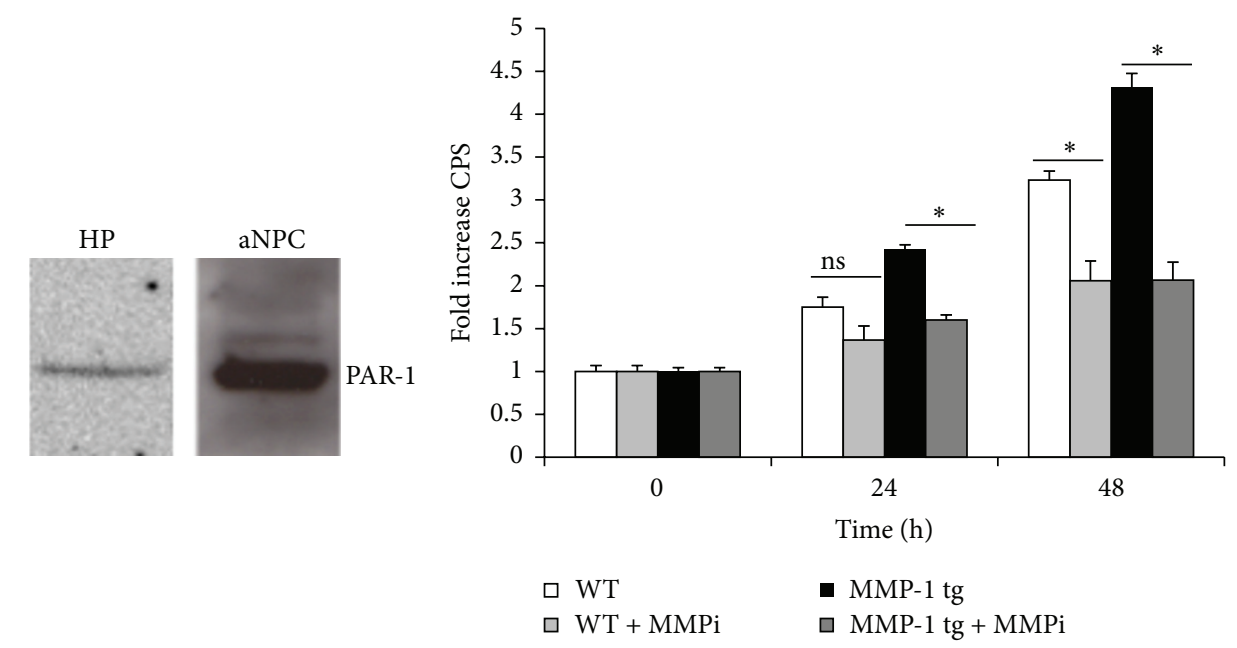

(a)

(b)

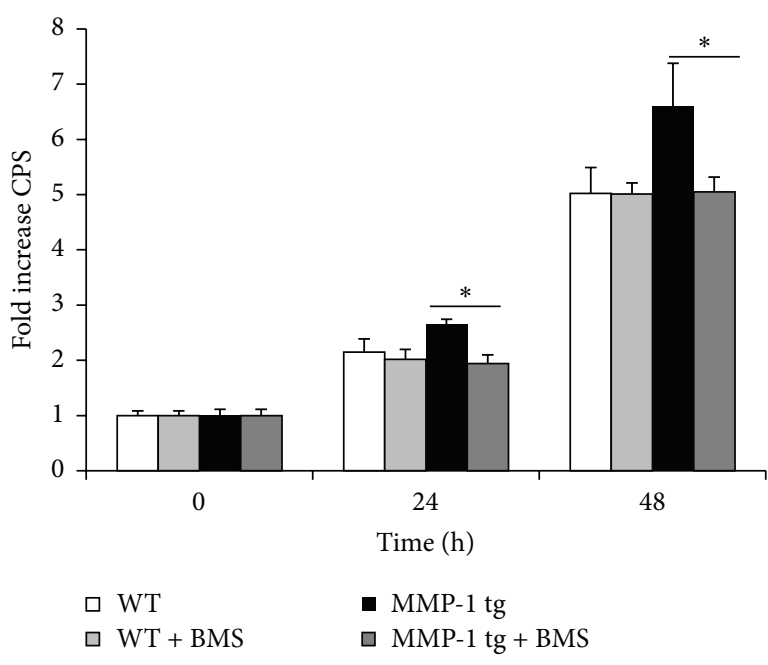

(c)

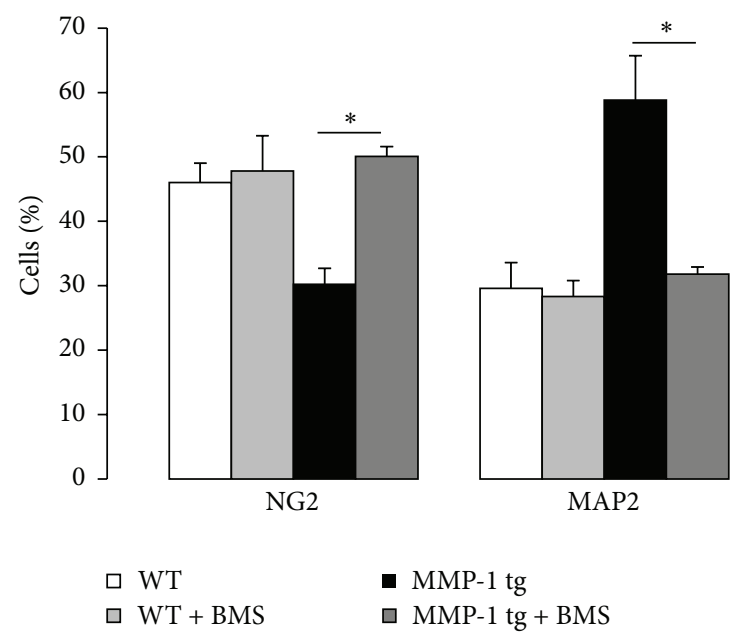

(d)

FIGURE 3: MMP-1 tg-associated changes in aNPC proliferation and differentiation are PAR-1 dependent. (a) Western blot analysis for PAR-1 expression in hippocampal (HP) and WT aNPC lysates. A single band at the expected molecular weight (46 kDa) is detected. (b) Effect of an MMP inhibitor on the proliferation rate of WT and tg cultures. $8 \mu \mathrm{M}$ MMP inhibitor II (MMPi) significantly reduced aNPC proliferation in tg cultures at $24 \mathrm{~h}$ time point and in both WT and tg cultures at $48 \mathrm{~h}\left({ }^{*} p<0.05\right.$; ANOVA) (b). (c) Effect of a PAR-1 antagonist on the proliferation of WT and tg cultures. $1 \mu \mathrm{M}$ BMS-200261 (BMS) reduced proliferation of aNPCs from MMP-1 tg animal. (d) Effect of the PAR-1 antagonist on the neuronal and nonneuronal differentiation of WT and tg cultures. BMS $(1 \mu \mathrm{M})$ significantly reduced enhanced neuronal differentiation and counteracted reduction in $\mathrm{NG}^{+}$cells in tg cultures $\left({ }^{*} p<0.05\right.$; ANOVA).

that MMP activity contributes to enhanced proliferation of tg aNPCs, we tested a broad spectrum inhibitor of MMP activity (MMP inhibitor II, MMPi, $8 \mu \mathrm{M}$ ) for its potential to inhibit this effect. As shown in Figure 3(b), inhibition of MMP activity significantly reduced aNPC proliferation in tg cultures at $24 \mathrm{~h}$ time point $\left({ }^{*} p<0.05\right.$; ANOVA $)$ and significantly reduced proliferation in both WT and tg cultures at $48 \mathrm{~h}\left({ }^{*} p<0.05\right.$; ANOVA). Fold change is shown (baseline CPS values were 71,863 $\pm 11,369$ for WT and $77,744 \pm 7898$ for MMP-1 tg). We next examined a PAR-1 antagonist for its potential to inhibit enhanced proliferation of aNPCs from MMP-1 tg animals. The PAR-1 antagonist BMS-200261 (BMS), used at $1 \mu \mathrm{M}$ concentration, significantly reduced proliferation of tg-derived aNPCs, and not that of
WT cultures, at $24 \mathrm{~h}$ and $48 \mathrm{~h}$ time points (Figure $3(\mathrm{c})$ ). Fold change is again shown (baseline CPS values were 34,496 \pm 2,971 for WT and 34,872 $\pm 3,962$ for MMP-1 tg). In parallel, as shown in Figure 3(d), the same treatment abolished increased neuronal differentiation in tg-derived aNPC $\left({ }^{*} p<0.05\right.$; ANOVA). Moreover, BMS counteracted the reduction in the percentage of $\mathrm{NG}_{2}{ }^{+}$oligodendrocyte precursors observed in tg cultures (Figure 3(d)) $\left({ }^{*} p<0.05\right.$; ANOVA). No significant effect was elicited by BMS on both neuronal and nonneuronal populations in WT-derived aNPC (Figure 3(d)). In addition, cell survival was not affected by the compound (mean percentage \pm SD of viable cells: WT: $94.9 \pm 1$; WT + BMS: $95.6 \pm 1.2$; MMP- 1 tg: $95.6 \pm 1.4$; MMP- 1 tg + BMS: 95.9 \pm 1.6 , ANOVA). 


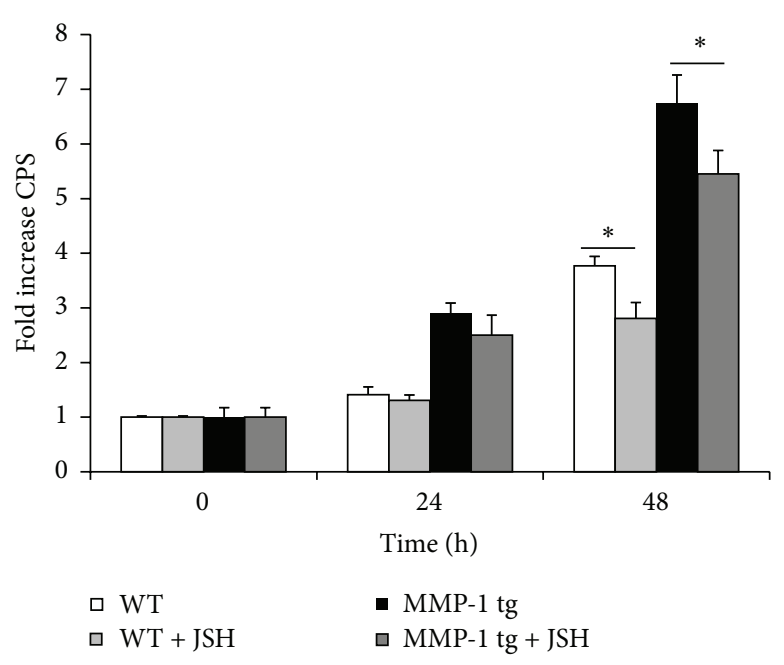

(a)

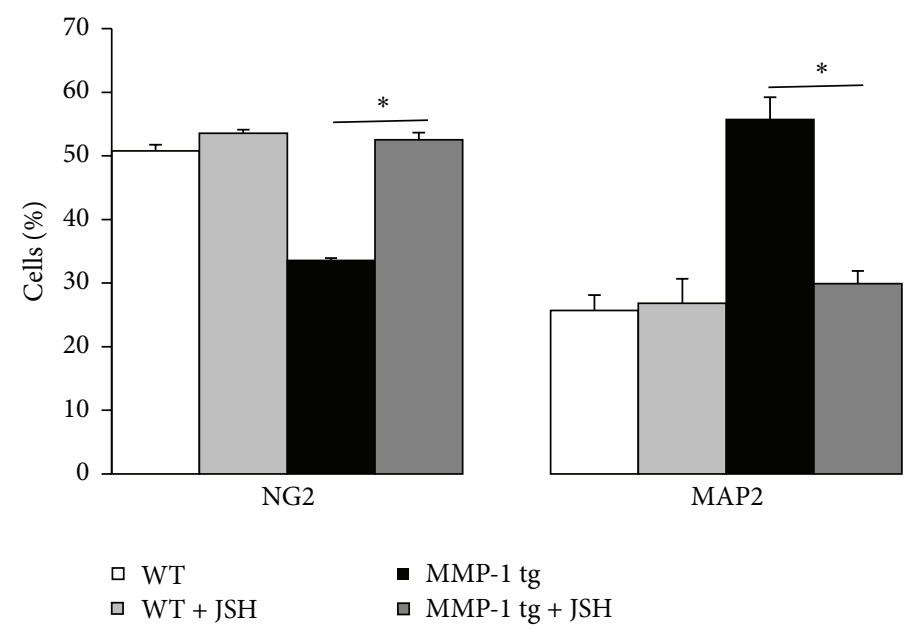

(b)

FIGURE 4: The nuclear translocation of NF- $\kappa$ B is required for MMP-1 tg associated changes in NPC proliferation and differentiation. (a) Effect of JSH-23 on aNPC proliferation in WT and tg cultures. The drug, tested at $3 \mu \mathrm{M}$, reduced proliferation of aNPCs in both WT and tg cultures at $48 \mathrm{~h}$. (b) Effect of JSH-23 on aNPC differentiation in WT and tg cultures. $3 \mu \mathrm{M}$ JSH did counteract enhanced neuronal differentiation $\left({ }^{*} p<0.05\right.$; ANOVA $)$ and reduced NG2 ${ }^{+}$cell number $\left({ }^{*} p<0.05\right.$; ANOVA $)$ in tg cultures, with no effect on WT aNPC.

3.4. The Nuclear Translocation of NF- $\kappa B$ p65 Is Required for MMP-1 tg Associated Changes in NPC Proliferation and Neuronal Differentiation. Previous studies have linked PAR1 to enhanced NF- $\kappa \mathrm{B}$ dependent transcription of genes including VEGF. Moreover, published studies suggest that NF- $\kappa$ B signaling is important to AHN [29-31]. We therefore tested an inhibitor of NF- $\kappa$ B signaling for its effects on NPC proliferation and differentiation in WT and tg cultures. As shown in Figure 4(a), the inhibitor of NF- $\kappa \mathrm{B}$ p65 nuclear translocation JSH-23 (JSH, $3 \mu \mathrm{M}$ ) reduced proliferation of aNPCs in both WT and tg cultures at $48 \mathrm{~h}$. Fold change is shown (baseline CPS data values were 23,239 \pm 532 for WT and 24,129 \pm 4202 for MMP- 1 tg). Moreover, JSH significantly reduced enhanced neuronal differentiation in tg-derived but not wt-derived cultures $\left({ }^{*} p<0.05\right.$; ANOVA). In parallel, JSH also counteracted the reduction in the percentage of oligodendrocyte precursors in tg-derived and not wt-derived aNPC (Figure $4(\mathrm{~b}))\left({ }^{*} p<0.05\right.$; ANOVA). Of note, JSH did not have a significant effect on MMP-1 levels in tg cultures (data not shown) and did not reduce cell survival in WT or tg cultures (mean percentage \pm SD of viable cells: WT: $96.8 \pm$ 0.97; WT + JSH: $96.5 \pm 1.24$; MMP-1 tg: $95.8 \pm 0.7$; MMP-1 tg + JSH: $95.3 \pm 0.9$, ANOVA).

3.5. SOX2 Positive NPCs Are Increased in the Hippocampus of MMP-1 tg Animals. Sequential actions of specific transcription factors are critical to proper development of AHN [32]. SOX2 is a transcription factor expressed in undifferentiated progenitor cells. It is a major mediator of Notch signaling and it is important to maintenance of the precursor pool in the adult subgranular zone (SGZ) of the DG [32-34]. We therefore examined SOX2 positive cell number in WT and MMP-1 tg hippocampus as a proxy for differences in the size

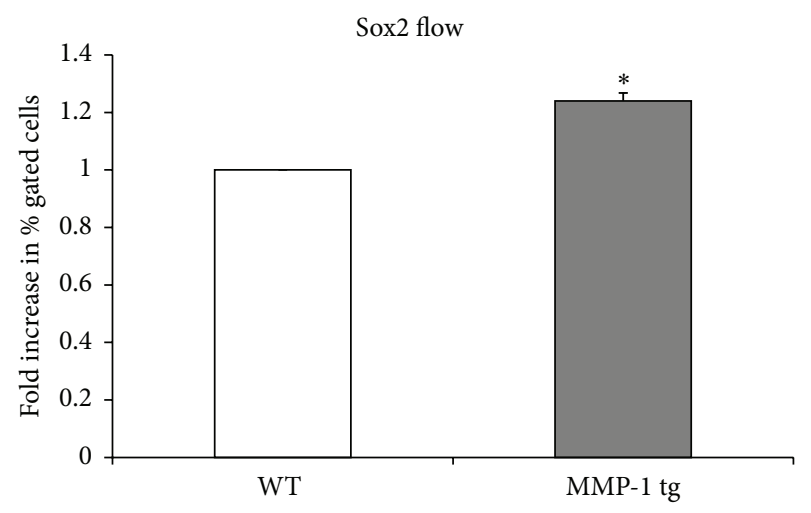

FIGURE 5: SOX2 positive NPCs are increased in the hippocampus of MMP-1 tg animals, compared to WT mice. Flow cytometric analysis of cell suspensions from murine hippocampus shows that the percentage of $\mathrm{SOX}^{+}$cells is significantly increased in tg compared to WT mice ( $n=4$ hippocampi per group with duplicate samples run in each of two separate experiments; ${ }^{*} p<0.05$, ANOVA).

of this pool. As shown in Figure 5, flow cytometric analysis of cell suspensions from the hippocampus suggests that the percentage of SOX2 positive cells is increased in MMP-1 tg compared to WT mice ( $n=4$ hippocampi per group with duplicate samples run in each of two separate experiments; $\left.{ }^{*} p<0.05\right)$.

\section{Discussion}

AHN is important to pattern separation and cognitive flexibility $[2,3]$. It may also play a role in mood regulation [35]. Consistent with this possibility, cancer treatments that 
ablate AHN are associated with depression and anxiety [35, 36]. Moreover, AHN is necessary for serotonin reuptake inhibitors to prevent behavioural correlates of depression in animal models [37, 38].

MMPs are increasingly recognized as important effectors of neuronal plasticity in both physiological and pathological settings [39-43]. In the adult brain, these enzymes are critical to selected forms of synaptic plasticity and recent work suggests they may also contribute to AHN. For example, broad spectrum MMPi reduces proliferation and neuronal differentiation of neural stem cells from umbilical cord blood [44], and MMP activity contributes to stroke-induced neurogenesis [45]. Moreover, MMP-2 expression is detected in SOX2 immunopositive progenitor cells in the teleost fish, while siRNA to select MMPs abrogates enhanced neurogenesis in a murine model of intracerebral haemorrhage [46, 47]. Unanswered questions include those related to whether specific MMPs are sufficient to drive enhanced neurogenesis and those related to the mechanisms through which MMPs can enhance aNPC proliferation and/or differentiation.

In the present study, we show that overexpression of MMP-1 is sufficient to drive increased proliferation of aNPCs in vitro. Moreover, these data were corroborated by flow cytometry data demonstrating the presence of a significantly higher number of SOX2 positive cells in hippocampi of MMP-1 tg mice when compared to their WT counterpart. In vitro, MMP-1 overexpression also stimulates enhanced neuronal fate specification of progenitors, with no changes in astrocyte generation and a parallel reduction in the number of $\mathrm{NG}^{+}$oligodendrocyte precursors. Importantly, increased proliferation and enhanced neuronal differentiation of MMP1 tg-derived aNPC could be abrogated by an inhibitor of PAR1 signaling. As compared to the broad spectrum MMPi, which reduced proliferation of both WT and tg aNPCs, a PAR-1 antagonist did not substantially influence this endpoint in WT cultures. Differential effects of MMP versus PAR antagonists could relate to release of murine-encoded MMPs from tg cultures that act through PAR-1 independent mechanisms including proneurotrophin conversion [48]. Broad spectrum inhibition of MMP activity may thus influence neurogenesis at many levels and differences in both cell proliferation and survival should be considered.

We also show that an inhibitor of NF- $\kappa$ B p 65 nuclear translocation, JSH-23, reduces neuronal differentiation in a MMP-1 tg selective manner. This is consistent with prior work in which JSH-23 did not diminish neuronal differentiation when administered in isolation but did diminish enhanced neuronal differentiation in response to a drug that could activate the NF- $\kappa$ B pathway and in parallel increased hippocampal neurogenesis both in vitro and in vivo [31]. Though additional studies will be necessary to determine whether MMP-1 is a relatively direct effector of NF- $\kappa$ B signaling, data herein are consistent with the possibility that NF- $\kappa \mathrm{B}$ activation is increased in tg-derived aNPCs.

PAR-1 stimulated effects on aNPC proliferation and differentiation are not without precedent. Previous studies with PAR-1 agonists that may enter the CNS with injury, including activated protein C (APC) and thrombin [49-51], suggest this receptor may be important to AHN which follows ischemic or hemorrhagic insult. APC analogues can also promote neurogenesis in rodents following focal ischemic stroke [52]. Effects of APC and thrombin on hippocampal neurogenesis may, however, be most relevant to select pathological states in which blood brain barrier damage occurs. Moreover, as compared to thrombin and APC, MMP-1 generates a unique peptide agonist with the potential to stimulate distinct intracellular signaling events [12]. Thrombin, APC, and MMP-1 cleave PAR-1 at arginine 41, arginine 41 or 46 , and aspartate 39, respectively. The MMP-1 generated tethered ligand is thus longer by at least two amino acids. This is important in that small differences in GPCR agonist structure can alter receptor localization, $G$ protein coupling, and the duration of signaling [53]. Consistent with this, Blackburn and Brinckerhoff have shown that equimolar concentrations $(5 \mathrm{nM})$ of MMP-1 and thrombin have differential effects on human microvessel endothelial cells. MMP-1 stimulates relatively long-lived increases in p 38 and MEK/ERK signaling and gene expression analysis also showed differences as a function of agonist [12]. Intriguingly, MMP-1 stimulated a 3fold increase in the expression of the proneurogenic molecule VEGFA, while there was no increase of VEGFA expression in response to thrombin $[12,54]$. Additional work has linked PAR-1 signaling to enhanced release of MMP-9 [55], an additional potential effector of neuronal differentiation [45].

With respect to future studies and the question of the MMP-1/PAR-1 axis in regulating AHN under physiological and/or pathological conditions, it should be noted that MMP1 expression may be increased in association with select physiological and pathological stimuli relevant to neurogenesis. Future studies will be necessary to determine whether PAR1 activating MMPs are important for neurogenesis in the background of environmental enrichment and/or antidepressant medication. MMP-1 levels may also be increased in disorders of the central nervous system characterized by glial activation. Such disorders include fragile X syndrome [56], a condition in which enhanced neurogenesis may be observed [57]. Given that the MMP-1 promoter is driven by transcription factors including AP-1, which are strongly modulated by excitatory neurotransmission [58], future studies might also address MMP- 1 dependent neurogenesis as a function of seizure activity or electroconvulsive therapy.

\section{Conclusions}

Herein we suggest that the MMP-1/PAR-1 axis can significantly impact both the proliferation and differentiation of aNPCs derived from hippocampus. Additional experiments may be warranted to determine whether this axis contributes to neurogenesis-related behaviors including cognitive flexibility and responsiveness to antidepressant therapy. Future studies might also examine the relevance of this axis to postinjury neurogenesis.

\section{Conflict of Interests}

The authors declare that there is no conflict of interests regarding the publication of this paper. 


\section{Acknowledgments}

This work was supported in part by NIH/National Institute of Neurological Disorders and Stroke T32NS041218 (M.A.) and R01NS083410 and R21NS052580 (K.C.). The authors would like to thank the Flow Cytometry and Cell Sorting Shared Resource (Dr. Karen Creswell) at Georgetown University Medical Center, which is partially supported by NIH/NCI Grant no. P30-CA051008.

\section{References}

[1] G.-L. Ming and H. Song, "Adult neurogenesis in the mammalian brain: significant answers and significant questions," Neuron, vol. 70, no. 4, pp. 687-702, 2011.

[2] A. Garthe, Z. Huang, L. Kaczmarek, R. K. Filipkowski, and G. Kempermann, "Not all water mazes are created equal: cyclin D2 knockout mice with constitutively suppressed adult hippocampal neurogenesis do show specific spatial learning deficits," Genes, Brain and Behavior, vol. 13, no. 4, pp. 357-364, 2014.

[3] A. A. Swan, J. E. Clutton, P. K. Chary, S. G. Cook, G. G. Liu, and M. R. Drew, "Characterization of the role of adult neurogenesis in touch-screen discrimination learning," Hippocampus, vol. 24, no. 12, pp. 1581-1591, 2014.

[4] K. Hisaoka-Nakashima, K. Miyano, C. Matsumoto et al., "Tricyclic antidepressant amitriptyline-induced glial cell linederived neurotrophic factor production involves pertussis toxin-sensitive $\mathrm{G} \alpha_{\mathrm{i} / \mathrm{o}}$ activation in astroglial cells," The Journal of Biological Chemistry, vol. 290, no. 22, pp. 13678-13691, 2015.

[5] M. Asahi, K. Asahi, J.-C. Jung, G. J. Del Zoppo, M. E. Fini, and E. H. Lo, "Role for matrix metalloproteinase 9 after focal cerebral ischemia: effects of gene knockout and enzyme inhibition with BB-94," Journal of Cerebral Blood Flow and Metabolism, vol. 20, no. 12, pp. 1681-1689, 2000.

[6] W. Cao, J. Duan, X. Wang et al., "Early enriched environment induces an increased conversion of proBDNF to BDNF in the adult rat's hippocampus," Behavioural Brain Research, vol. 265, pp. 76-83, 2014.

[7] L. Wójcik-Stanaszek, J. Sypecka, P. Szymczak et al., “The potential role of metalloproteinases in neurogenesis in the gerbil hippocampus following global forebrain ischemia," PLoS ONE, vol. 6, no. 7, Article ID e22465, 2011.

[8] K.-S. Han, G. Mannaioni, C. E. Hamill et al., "Activation of protease activated receptor 1 increases the excitability of the dentate granule neurons of hippocampus," Molecular Brain, vol. 4, no. 1, article 32, 2011.

[9] U. J. Soh, M. R. Dores, B. Chen, and J. Trejo, "Signal transduction by protease-activated receptors," British Journal of Pharmacology, vol. 160, no. 2, pp. 191-203, 2010.

[10] R. Kaufmann and M. D. Hollenberg, "Proteinase-activated receptors (PARs) and calcium signaling in cancer," Advances in Experimental Medicine and Biology, vol. 740, pp. 979-1000, 2012.

[11] F. Striggow, M. Riek-Burchardt, A. Kiesel et al., "Four different types of protease-activated receptors are widely expressed in the brain and are up-regulated in hippocampus by severe ischemia," European Journal of Neuroscience, vol. 14, no. 4, pp. 595-608, 2001.

[12] J. S. Blackburn and C. E. Brinckerhoff, "Matrix metalloproteinase- 1 and thrombin differentially activate gene expression in endothelial cells via PAR-1 and promote angiogenesis," The American Journal of Pathology, vol. 173, no. 6, pp. 1736-1746, 2008.

[13] R. Mazor, T. Alsaigh, H. Shaked et al., "Matrix metalloproteinase-1-mediated up-regulation of vascular endothelial growth factor-2 in endothelial cells," The Journal of Biological Chemistry, vol. 288, no. 1, pp. 598-607, 2013.

[14] A. Boire, L. Covic, A. Agarwal, S. Jacques, S. Sherifi, and A. Kuliopulos, "PAR1 is a matrix metalloprotease-1 receptor that promotes invasion and tumorigenesis of breast cancer cells," Cell, vol. 120, no. 3, pp. 303-313, 2005.

[15] G. Koukos, L. Sevigny, P. Zhang, L. Covic, and A. Kuliopulos, "Serine and metalloprotease signaling through PAR1 in arterial thrombosis and vascular injury," IUBMB Life, vol. 63, no. 6, pp. 412-418, 2011.

[16] C. M. P. Vos, L. Sjulson, A. Nath et al., "Cytotoxicity by matrix metalloprotease-1 in organotypic spinal cord and dissociated neuronal cultures," Experimental Neurology, vol. 163, no. 2, pp. 324-330, 2000.

[17] M. D. Ross, A. L. Wekesa, J. P. Phelan, and M. Harrison, "Resistance exercise increases endothelial progenitor cells and angiogenic factors," Medicine and Science in Sports and Exercise, vol. 46, no. 1, pp. 16-23, 2014.

[18] K. Chen, D. Li, X. Zhang, P. L. Hermonat, and J. L. Mehta, "Anoxia-reoxygenation stimulates collagen type-1 and MMP1 expression in cardiac fibroblasts: modulation by the PPAR- $\gamma$ ligand pioglitazone," Journal of Cardiovascular Pharmacology, vol. 44, no. 6, pp. 682-687, 2004.

[19] V. N. Ierusalimsky and P. M. Balaban, "Type 1 metalloproteinase is selectively expressed in adult rat brain and can be rapidly upregulated by kainate," Acta Histochemica, vol. 115, no. 8, pp. 816826, 2013.

[20] A. D. R. Garcia, N. B. Doan, T. Imura, T. G. Bush, and M. V. Sofroniew, "GFAP-expressing progenitors are the principal source of constitutive neurogenesis in adult mouse forebrain," Nature Neuroscience, vol. 7, no. 11, pp. 1233-1241, 2004.

[21] M. Balbín, A. Fueyo, V. Knäuper et al., "Identification and enzymatic characterization of two diverging murine counterparts of human interstitial collagenase (MMP-1) expressed at sites of embryo implantation," The Journal of Biological Chemistry, vol. 276, no. 13, pp. 10253-10262, 2001.

[22] S. L. Tressel, N. C. Kaneider, S. Kasuda et al., "A matrix metalloprotease-PAR1 system regulates vascular integrity, systemic inflammation and death in sepsis," EMBO Molecular Medicine, vol. 3, no. 7, pp. 370-384, 2011.

[23] R. F. Foronjy, Y. Okada, R. Cole, and J. D’Armiento, "Progressive adult-onset emphysema in transgenic mice expressing human MMP-1 in the lung," The American Journal of Physiology-Lung Cellular and Molecular Physiology, vol. 284, no. 5, pp. L727-L737, 2003.

[24] H. E. Kim, S. S. Dalal, E. Young, M. J. Legato, M. L. Weisfeldt, and J. D'Armiento, "Disruption of the myocardial extracellular matrix leads to cardiac dysfunction," The Journal of Clinical Investigation, vol. 106, no. 7, pp. 857-866, 2000.

[25] M. Brenner, W. C. Kisseberth, Y. Su, F. Besnard, and A. Messing, "GFAP promoter directs astrocyte-specific expression in transgenic mice," The Journal of Neuroscience, vol. 14, no. 3, part 1, pp. 1030-1037, 1994.

[26] V. Meneghini, V. Bortolotto, M. T. Francese et al., "Highmobility group box-1 protein and $\beta$-amyloid oligomers promote neuronal differentiation of adult hippocampal neural progenitors via receptor for advanced glycation end products/nuclear 
factor- $\kappa \mathrm{B}$ axis: relevance for Alzheimer's disease," Journal of Neuroscience, vol. 33, no. 14, pp. 6047-6059, 2013.

[27] N. Maggio, C. Cavaliere, M. Papa, I. Blatt, J. Chapman, and M. Segal, "Thrombin regulation of synaptic transmission: implications for seizure onset," Neurobiology of Disease, vol. 50, no. 1, pp. 171-178, 2013.

[28] H. Guo, Z. Zhao, Q. Yang et al., "An activated protein C analog stimulates neuronal production by human neural progenitor cells via a PAR1-PAR3-S1PR1-Akt pathway," The Journal of Neuroscience, vol. 33, no. 14, pp. 6181-6190, 2013.

[29] S. Denis-Donini, A. Caprini, C. Frassoni, and M. Grilli, "Members of the NF- $\kappa \mathrm{B}$ family expressed in zones of active neurogenesis in the postnatal and adult mouse brain," Developmental Brain Research, vol. 154, no. 1, pp. 81-89, 2005.

[30] S. Denis-Donini, A. Dellarole, P. Crociara et al., "Impaired adult neurogenesis associated with short-term memory defects in NF-kappaB p50-deficient mice," Journal of Neuroscience, vol. 28, no. 15, pp. 3911-3919, 2008.

[31] B. Cuccurazzu, V. Bortolotto, M. M. Valente et al., "Upregulation of mGlu2 receptors via NF- $\kappa$ B p 65 acetylation is involved in the proneurogenic and antidepressant effects of acetyl-Lcarnitine," Neuropsychopharmacology, vol. 38, no. 11, pp. 2220 2230, 2013.

[32] G.-L. Ming and H. Song, "Adult neurogenesis in the mammalian central nervous system," Annual Review of Neuroscience, vol. 28, pp. 223-250, 2005.

[33] O. Ehm, C. Göritz, M. Covic et al., "RBPJkappa-dependent signaling is essential for long-term maintenance of neural stem cells in the adult hippocampus," The Journal of Neuroscience, vol. 30, no. 41, pp. 13794-13807, 2010.

[34] R. Favaro, M. Valotta, A. L. M. Ferri et al., "Hippocampal development and neural stem cell maintenance require Sox2dependent regulation of Shh," Nature Neuroscience, vol. 12, no. 10, pp. 1248-1256, 2009.

[35] B. R. Miller and R. Hen, "The current state of the neurogenic theory of depression and anxiety," Current Opinion in Neurobiology, vol. 30, pp. 51-58, 2015.

[36] G. P. Dias, R. Hollywood, M. C. do Nascimento Bevilaqua et al., "Consequences of cancer treatments on adult hippocampal neurogenesis: implications for cognitive function and depressive symptoms," Neuro-Oncology, vol. 16, no. 4, pp. 476-492, 2014.

[37] L. Santarelli, M. Saxe, C. Gross et al., "Requirement of hippocampal neurogenesis for the behavioral effects of antidepressants," Science, vol. 301, no. 5634, pp. 805-809, 2003.

[38] T. D. Perera, A. J. Dwork, K. A. Keegan et al., "Necessity of hippocampal neurogenesis for the therapeutic action of antidepressants in adult Nonhuman primates," PLOS ONE, vol. 6, no. 4, Article ID e17600, 2011.

[39] G. W. Huntley, "Synaptic circuit remodelling by matrix metalloproteinases in health and disease," Nature Reviews Neuroscience, vol. 13, no. 11, pp. 743-757, 2012.

[40] P. Sonderegger and K. Matsumoto-Miyai, "Activity-controlled proteolytic cleavage at the synapse," Trends in Neurosciences, vol. 37, no. 8, pp. 413-423, 2014.

[41] A. C. W. Smith, Y. M. Kupchik, M. D. Scofield et al., "Synaptic plasticity mediating cocaine relapse requires matrix metalloproteinases," Nature Neuroscience, vol. 17, no. 12, pp. 1655-1657, 2014.
[42] T. E. Brown, M. R. Forquer, D. L. Cocking, H. T. Jansen, J. W. Harding, and B. A. Sorg, "Role of matrix metalloproteinases in the acquisition and reconsolidation of cocaine-induced conditioned place preference," Learning and Memory, vol. 14, no. 3, pp. 214-223, 2007.

[43] P. Michaluk, M. Wawrzyniak, P. Alot et al., "Influence of matrix metalloproteinase MMP-9 on dendritic spine morphology," Journal of Cell Science, vol. 124, no. 19, pp. 3369-3380, 2011.

[44] L. Wojcik, A. Sawicka, S. Rivera, and T. Zalewska, "Neurogenesis in gerbil hippocampus following brain ischemia: focus on the involvement of metalloproteinases," Acta Neurobiologiae Experimentalis, vol. 69, no. 1, pp. 52-61, 2009.

[45] B. Z. Barkho, A. E. Munoz, X. Li, L. Li, L. A. Cunningham, and X. Zhao, "Endogenous matrix metalloproteinase (MMP)-3 and MMP-9 promote the differentiation and migration of adult neural progenitor cells in response to chemokines," Stem Cells, vol. 26, no. 12, pp. 3139-3149, 2008.

[46] C. Lei, S. Lin, C. Zhang et al., "Activation of cerebral recovery by matrix metalloproteinase- 9 after intracerebral hemorrhage," Neuroscience, vol. 230, pp. 86-93, 2013.

[47] R. F. Sîrbulescu, I. Ilieş, and G. K. Zupanc, "Matrix metalloproteinase-2 and -9 in the cerebellum of teleost fish: functional implications for adult neurogenesis," Molecular and Cellular Neuroscience, vol. 68, pp. 9-23, 2015.

[48] R. Lee, P. Kermani, K. K. Teng, and B. L. Hempstead, "Regulation of cell survival by secreted proneurotrophins," Science, vol. 294, no. 5548, pp. 1945-1948, 2001.

[49] S. Yang, S. Song, Y. Hua, T. Nakamura, R. F. Keep, and G. $\mathrm{Xi}$, "Effects of thrombin on neurogenesis after intracerebral hemorrhage," Stroke, vol. 39, no. 7, pp. 2079-2084, 2008.

[50] Y. Wang, Z. Zhao, N. Chow, T. Ali, J. H. Griffin, and B. V. Zlokovic, "Activated protein $\mathrm{C}$ analog promotes neurogenesis and improves neurological outcome after focal ischemic stroke in mice via protease activated receptor 1," Brain Research, vol. 1507, pp. 97-104, 2013.

[51] M. Thiyagarajan, J. A. Fernández, S. M. Lane, J. H. Griffin, and B. V. Zlokovic, "Activated protein $\mathrm{C}$ promotes neovascularization and neurogenesis in postischemic brain via protease-activated receptor 1," Journal of Neuroscience, vol. 28, no. 48, pp. 1278812797, 2008.

[52] J. H. Griffin, B. V. Zlokovic, and L. O. Mosnier, "Activated protein C: biased for translation," Blood, vol. 125, no. 19, pp. 2898-2907, 2015.

[53] J.-P. Vilardaga, F. G. Jean-Alphonse, and T. J. Gardella, "Endosomal generation of cAMP in GPCR signaling," Nature Chemical Biology, vol. 10, no. 9, pp. 700-706, 2014.

[54] N. M. Fournier, B. Lee, M. Banasr, M. Elsayed, and R. S. Duman, "Vascular endothelial growth factor regulates adult hippocampal cell proliferation through MEK/ERK- and PI3K/Aktdependent signaling," Neuropharmacology, vol. 63, no. 4, pp. 642-652, 2012.

[55] A. Agarwal, L. Covic, L. M. Sevigny et al., "Targeting a metalloprotease-PAR1 signaling system with cell-penetrating pepducins inhibits angiogenesis, ascites, and progression of ovarian cancer," Molecular Cancer Therapeutics, vol. 7, no. 9, pp. 2746-2757, 2008.

[56] C. J. Yuskaitis, E. Beurel, and R. S. Jope, "Evidence of reactive astrocytes but not peripheral immune system activation in a mouse model of Fragile X syndrome," Biochimica et Biophysica Acta: Molecular Basis of Disease, vol. 1802, no. 11, pp. 1006-1012, 2010. 
[57] Y. Luo, G. Shan, W. Guo et al., "Fragile X mental retardation protein regulates proliferation and differentiation of adult neural stem/progenitor cells," PLoS Genetics, vol. 6, no. 4, 2010.

[58] D. Konopka, D. Nowicka, R. K. Filipkowski, and L. Kaczmarek, "Kainate-evoked secondary gene expression in the rat hippocampus," Neuroscience Letters, vol. 185, no. 3, pp. 167-170, 1995. 

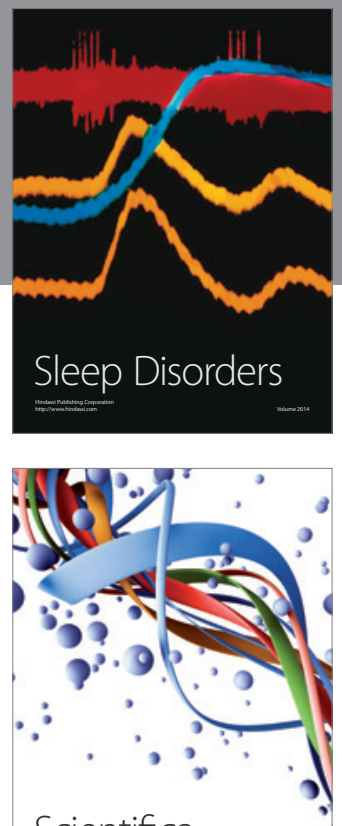

Scientifica
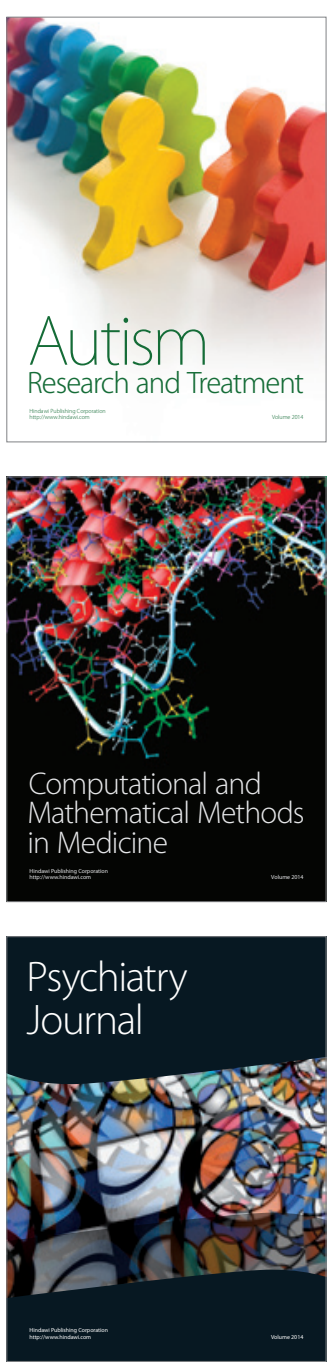
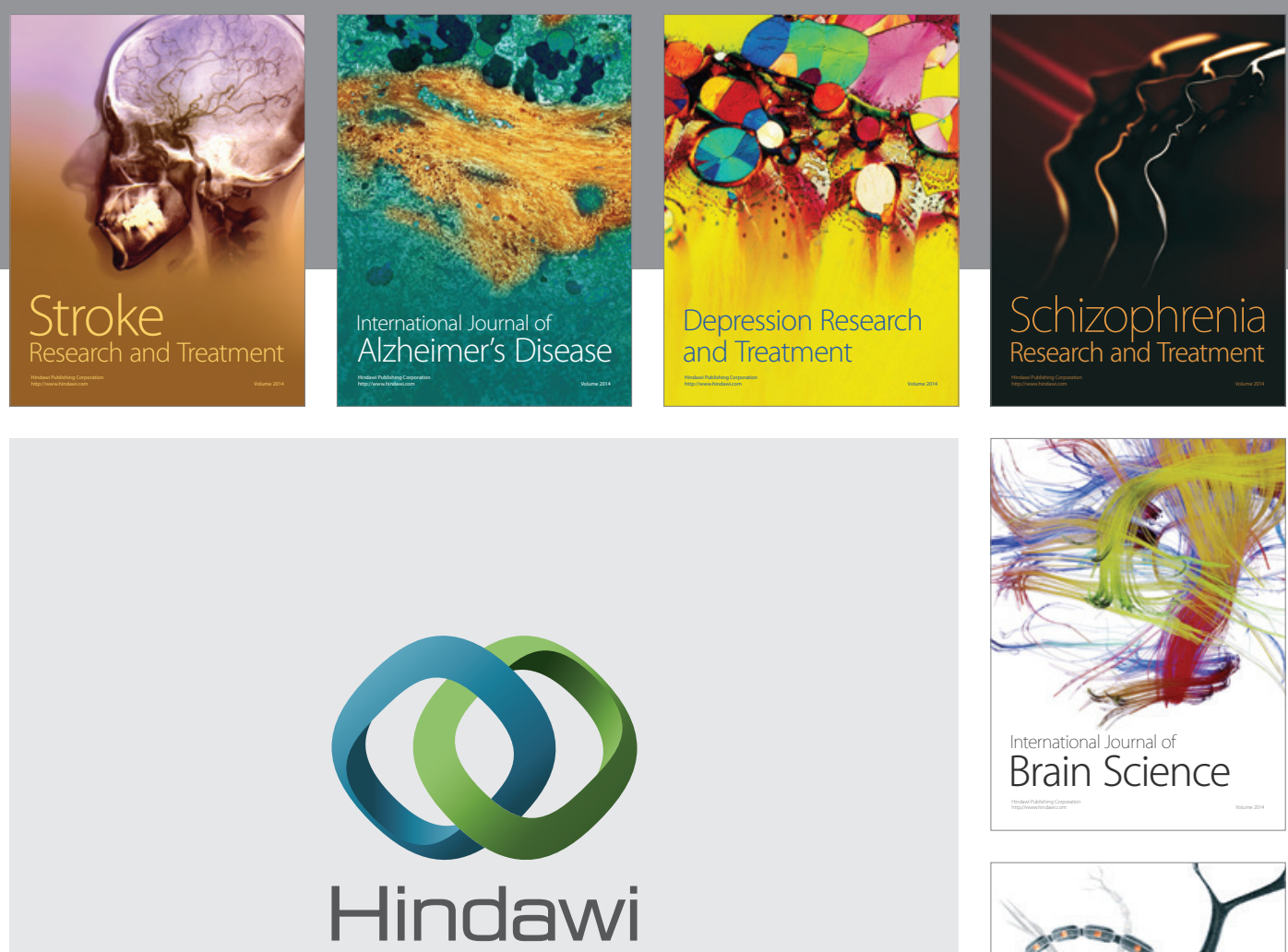

Submit your manuscripts at

http://www.hindawi.com
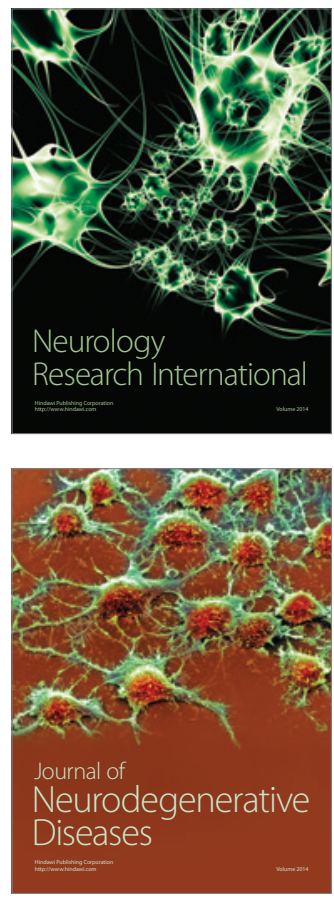

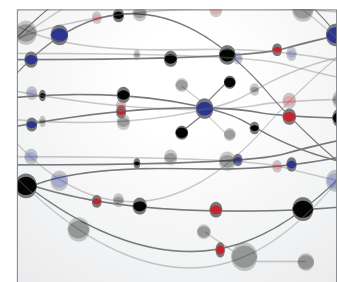

The Scientific World Journal
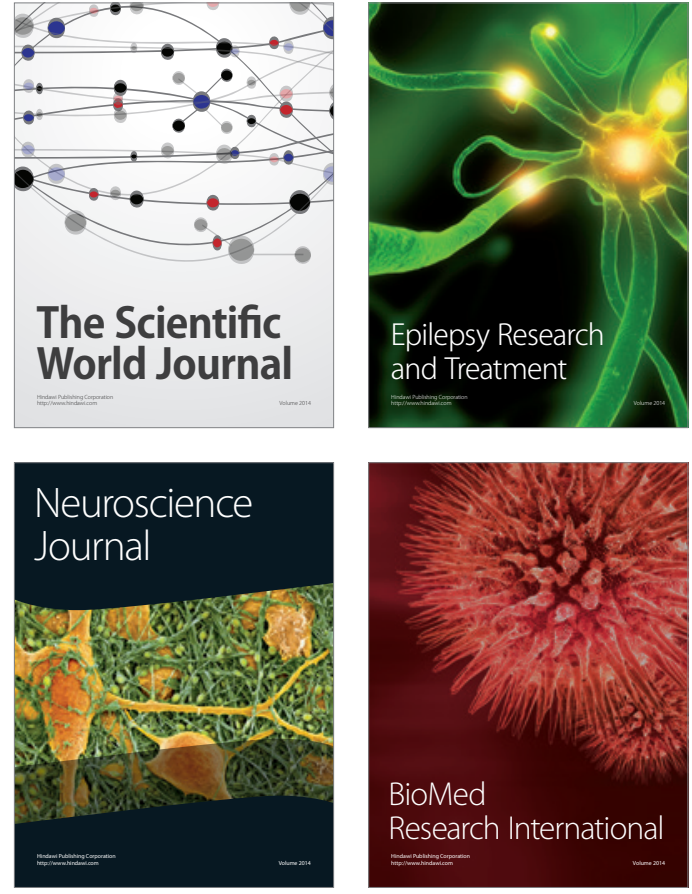

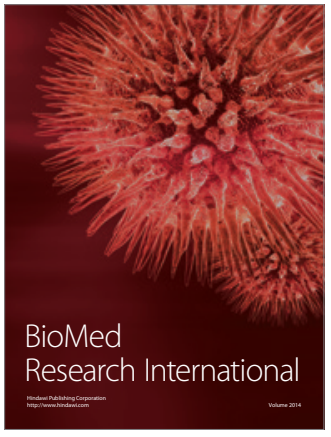

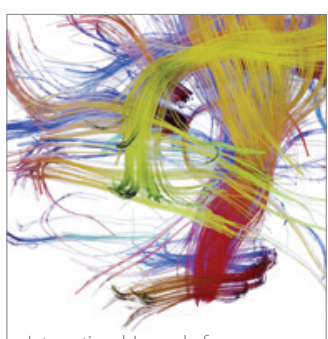

Brain Science

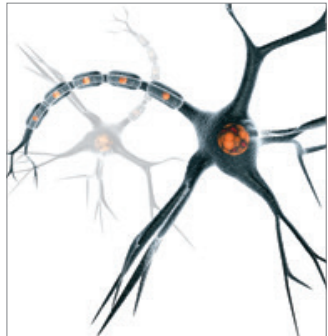

Neural Plasticity
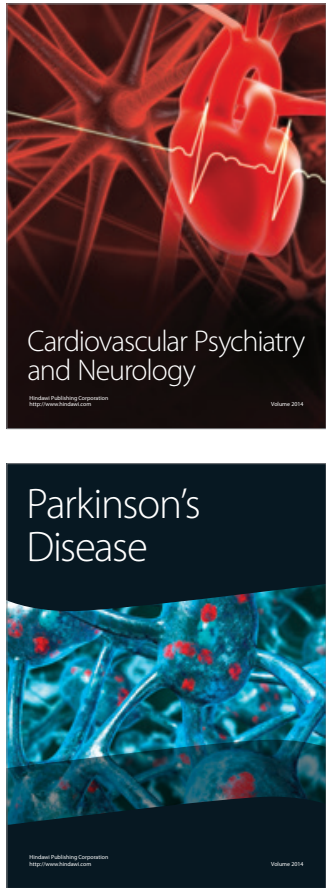